\title{
Apelin/APJ signaling promotes hypoxia-induced proliferation of endothelial progenitor cells via phosphoinositide-3 kinase/Akt signaling
}

\author{
JINGCHANG ZHANG ${ }^{1}$, QIMING LIU ${ }^{1}$, XINQUN HU $^{1}$, ZHENFEI FANG ${ }^{1}$, \\ FENG HUANG $^{2}$, LIANG TANG ${ }^{1}$ and SHENGHUA ZHOU ${ }^{1}$ \\ ${ }^{1}$ Department of Cardiology, The Second Xiangya Hospital of Central South University, Changsha, Hunan 410011; \\ ${ }^{2}$ Department of Cardiology, The First Affiliated Hospital of Guangxi Medical University, \\ Nanning, Guangxi 530021, P.R. China
}

Received August 16, 2014; Accepted April 30, 2015

DOI: $10.3892 / \mathrm{mmr} .2015 .3866$

\begin{abstract}
Endothelial progenitor cells (EPCs) can adhere to the endothelium at sites of hypoxia/ischemia and participate in the formation of novel vessels through differentiating into endothelial cells (ECs). Apelin is an endogenous ligand for the G protein-coupled receptor APJ, and apelin/APJ signaling has a role in cardiovascular function. The present study aimed to investigate the role of apelin/APJ signaling in the regulation of EPC proliferation under hypoxia. The results showed that hypoxia was able to induce EPC proliferation, accompanied with an upregulation of hypoxia-inducible factor (HIF)-1 $\alpha$ as well as apelin/APJ signaling. Further investigation indicated that siRNA-mediated knockdown of apelin or APJ expression attenuated the hypoxia-induced proliferation of EPCs, suggesting that apelin/APJ signaling has an important role in hypoxia-induced EPC proliferation. Moreover, the phosphoinositide-3 kinase (PI3K)/Akt signaling pathway was found to be involved in the apelin/APJ-mediated EPC proliferation under hypoxia. Based on these findings, the present study suggested that hypoxia-induced upregulation of HIF-1 $\alpha$ promotes the expression of apelin and APJ, which further activate the downstream PI3K/Akt signaling pathway, a key promoter of EPC proliferation. In conclusion, the present study highlighted the role of apelin/APJ in the regulation of EPC proliferation, and apelin/APJ may therefore serve as a potential target for the prevention of hypoxic ischemic injury.
\end{abstract}

Correspondence to: Professor Shenghua Zhou, Department of Cardiology, The Second Xiangya Hospital of Central South University, 139 People's Middle Road, Changsha, Hunan 410011, P.R. China

E-mail: xyzhoushenghua@163.com

Key words: proliferation, endothelial progenitor cells, hypoxia, apelin

\section{Introduction}

Oxidative stress-induced endothelial dysfunction is involved in ischemia/reperfusion injury, which may further cause fatal diseases, including coronary atherosclerosis caused by myocardial infarction and stroke $(1,2)$. It has been well-established that endothelial progenitor cells (EPCs) have a crucial role in angiogenesis of endothelial cells (ECs). They can adhere to the endothelium at sites of hypoxia/ischemia and participate in the formation of novel vessels through differentiating into ECs.

Hypoxia has been shown to have effects on multiple cellular biological processes of EPCs. For instance, hypoxia is able to induce the mobilization of EPCs from the bone marrow into the peripheral blood (3). Furthermore, hypoxia can induce endothelial cells to secret macrophage migration inhibitory factor (MIF), which may have a crucial role in the recruitment and migration of EPCs to hypoxic tissues (4). Lee et al (5) showed that hypoxia inhibited senescence of EPCs from aged individuals. The phosphoinositide-3 kinase (PI3K)/Akt signaling pathway has been found to have an essential role in the regulation of cellular survival, proliferation and migration. Moreover, the activity of the PI3K/Akt signaling pathway is closely associated with hypoxia-induced EPCs. Dai et al (6) showed that hypoxia was able to protect against serum withdrawal-induced EPC apoptosis, at least in part via the activation of the PI3K/Akt pathway.

Hypoxia-inducible factor- $1 \alpha$ (HIF-1 $\alpha$ ) is a key transcriptional factor, the expression of which can be markedly induced under hypoxia (7). Moreover, HIF-1 $\alpha$ has been shown to promote the proliferation and differentiation of EPCs. Jiang et al (8) reported that inhibition of HIF-1 $\alpha$ suppressed the differentiation of EPCs to ECs, as well as the expression of genes which promote neovascularization. However, the regulatory pattern of HIF-1 $\alpha$ under hypoxia in EPCs remains to be fully elucidated.

Apelin is an endogenous ligand for the $\mathrm{G}$ protein-coupled receptor APJ. The apelin/APJ pathway has a role in the regulation of diverse biological functions, including cardiovascular function, fluid homeostasis and insulin secretion, via activation 
of various tissue-specific signaling pathways (9-11). Recently, Ye et al (12) showed that the downregulation of the serum levels of apelin was associated with acute myocardial infarction-induced mobilization of EPCs, suggesting that apelin may be involved in the regulation of EPCs. Furthermore, apelin deficiency impaired sprouting of EPCs in ischemia-reperfusion injury (13). However, to the best of our knowledge, whether apelin has a role in the regulation of hypoxia-induced EPC proliferation as well as the underlying molecular mechanism have not been investigated, yet.

The present study mainly aimed to investigate the role of apelin/APJ signaling in hypoxia-induced EPC proliferation and the underlying molecular mechanisms were also investigated.

\section{Materials and methods}

Cell culture. EPCs were purchased from Nlunbio (Changsha, China). The EPCs were cultured in endothelial growth medium 2 (EGM-2) supplemented with $20 \%$ fetal bovine serum (FBS) both purchased from Life Technologies (Carlsbad, CA, USA) at $37^{\circ} \mathrm{C}$ in a humidified incubator containing $5 \%$ $\mathrm{CO}_{2}$. For performing cell proliferation analysis, LY294002 (Sigma Aldrich, St. Louis, MO, USA) and 740Y-P (Phoenix Pharmaceuticals, Belmont, CA, USA) were used to treat EPCs in different groups.

Hypoxia treatment of EPCs. Prior to hypoxia treatment, EPCs were cultured in serum-free EGM-2 at $37^{\circ} \mathrm{C}$ under $5 \% \mathrm{CO}_{2}$ for $12 \mathrm{~h}$. In the MTT assay (Sigma-Aldrich), EPCs were cultured in EGM-2 containing $20 \% \mathrm{FBS}$ at $37^{\circ} \mathrm{C}$ under $1 \% \mathrm{O}_{2}, 94 \% \mathrm{~N}_{2}$ and $5 \% \mathrm{CO}_{2}$ for $6,12,24$ and $48 \mathrm{~h}$, respectively.

Cell proliferation assay. The MTT assay was performed to determine cell proliferation. In brief, MTT $(10 \mathrm{mg} / \mathrm{ml})$ was added to the medium. After incubation for $4 \mathrm{~h}$, the reaction was terminated by removal of the supernatant and addition of $100 \mu$ l dimethyl sulfoxide (DMSO; Sigma-Aldrich) to dissolve the formazan product. After $30 \mathrm{~min}$, the optical density (OD) of each well was measured at $570 \mathrm{~nm}$ using a plate reader (ELx808; Bio-Tek Instruments, Winooski, WT, USA).

Reverse transcription quantitative polymerase chain reaction (RT-qPCR) analysis. TRIzol reagent (Life Technologies) was used to extract total RNA from EPCs, in accordance with the manufacturer's instructions. Total RNA was reverse transcribed into cDNA by using the PrimeScript RT reagent kit, according to the manufacturer's instructions (Takara Biotechnology Co., Ltd., Dalian, China). In brief, $5 \mu$ l total RNA was mixed with $0.15 \mu \mathrm{l}$ of $100 \mathrm{~mm}$ dNTPs (with dTTP), $1 \mu \mathrm{l}$ (50 units) reverse transcriptase, $1.5 \mu \mathrm{l}$ of $\mathrm{X} 10$ reverse transcription buffer, $0.19 \mu \mathrm{l}$ RNase inhibitor $(20 \mathrm{U} / \mu \mathrm{l})$, and nuclease-free $\mathrm{H}_{2} \mathrm{O}$ was added to obtain a final volume of $15 \mu \mathrm{l}$. Reverse transcription was performed at $16^{\circ} \mathrm{C}$ for $30 \mathrm{~min}$, followed by an incubation step at $42^{\circ} \mathrm{C}$ for $30 \mathrm{~min}$ and enzyme inactivation at $85^{\circ} \mathrm{C}$ for $5 \mathrm{~min}$. The $\mathrm{mRNA}$ expression levels were determined using SYBR Premix Ex Taq II, in accordance with the manufacturer's instructions (Takara Biotechnology Co., Ltd.). In brief, $0.2 \mu \mathrm{l}$ cDNA solution, $10 \mu \mathrm{l}$ PCR mix, $2 \mu$ l gene specific primer and $7.8 \mu 1$ nuclease-free $\mathrm{H}_{2} \mathrm{O}$ were mixed to obtain a final reaction volume of $20 \mu \mathrm{l}$. The

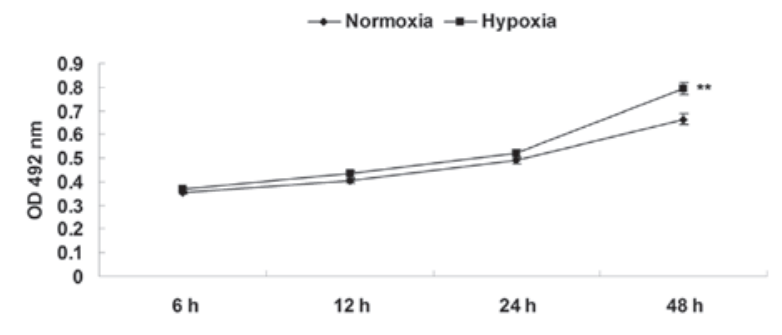

Figure 1. An MTT assay was performed to determine the cell proliferation of endothelial progenitor cells treated with normoxia or hypoxia for 6, 12, 24 and $48 \mathrm{~h}$. Values are expressed as the mean \pm standard deviation. ${ }^{* *} \mathrm{P}<0.01$, vs. normoxia. OD, optical density.

reaction conditions for PCR were: Pre-degeneration at $95^{\circ} \mathrm{C}$ for $1 \mathrm{~min}$ followed by 40 cycles of $95^{\circ} \mathrm{C}$ for $15 \mathrm{sec}, 60^{\circ} \mathrm{C}$ for $15 \mathrm{sec}$, and a final extension at $72^{\circ} \mathrm{C}$ for $1 \mathrm{~min}$. The specific primer pairs were as follows: HIF-1 $\alpha$ sense, 5'-GAACGT CGAAAAGAAAAGTCTCG-3' and anti-sense, 5'-CCTTAT CAAGATGCGAACTCACA-3'; apelin sense, 5'-GTCTCC TCCATAGATTGGTCTGC-3' and anti-sense, 5'-GGAATC ATCCAAACTACAGCCAG-3'; APJ sense, 5'-CTCTGGACC GTGTTTCGGAG-3' and anti-sense, 5'-GGTACGTGTAGG TAGCCCACA-3'; GAPDH (internal reference) sense, 5'-GGA GCGAGATCCCTCCAAAAT-3' and anti-sense, 5'-GGCTGT TGTCATACTTCTCATGG-3'. Primers were purchased from Shanghai Shenggong Co., Ltd. (Shanghai, China). Independent experiments were repeated three times. The relative expression of mRNA was analyzed using the $2^{-\Delta \Delta C t}$ method.

Transfection. Transfection was performed using Lipofectamine 2000 (Invitrogen Life Technologies, Carlsbad, CA, USA) in accordance with the manufacturer's instructions. All plasmids and siRNA were purchased from Nlunbio. Sequences or other information were not provided due to commercial confidentiality. Lipofectamine 2000, siRNA and plasmid were diluted with serum-free medium, respectively. The diluted Lipofectamine 2000 was added into the diluted siRNA or plasmid and incubated for $20 \mathrm{~min}$ at room temperature, and then added into the EPC suspension. Subsequently, EPCs were incubated at $37^{\circ} \mathrm{C}, 5 \% \mathrm{CO}_{2}$ for $6 \mathrm{~h}$. After that, the medium was replaced by Dulbecco's modified Eagle's medium with $10 \%$ FBS, and cultured for $24 \mathrm{~h}$ prior to the following assays.

Western blot analysis. Cells were solubilized in cold radioimmunoprecipitation assay lysis buffer (Sigma-Aldrich). Proteins were separated by $12 \%$ SDS-PAGE (Sigma-Aldrich) and transferred onto a polyvinylidene difluoride (PVDF) membrane (Life Technologies), which was then incubated with Tris-buffered saline with Tween 20 (TBST; Sigma-Aldrich) containing $5 \%$ skimmed milk at $4{ }^{\circ} \mathrm{C}$ for overnight. After that, the PVDF membrane was incubated with specific primary antibodies, rabbit polyclonal anti-Apelin (cat. no. ab59469; 1:100) and rabbit polyclonal anti-APJ (cat. no. ab84296; 1:100) and incubated at room temperature for $3 \mathrm{~h}$. After washing with TBST three times, the PVDF membrane was incubated with goat anti-rabbit IgG (cat. no. ab175734; 1:5,000, secondary antibodies at room temperature for $1 \mathrm{~h}$. All antibodies were purchased from Abcam (Cambridge, MA, USA). After washing with TBST three times, an enhanced chemiluminescence kit 
A

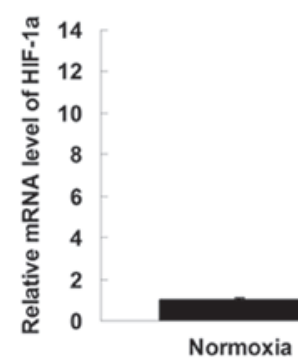

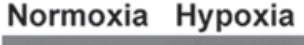
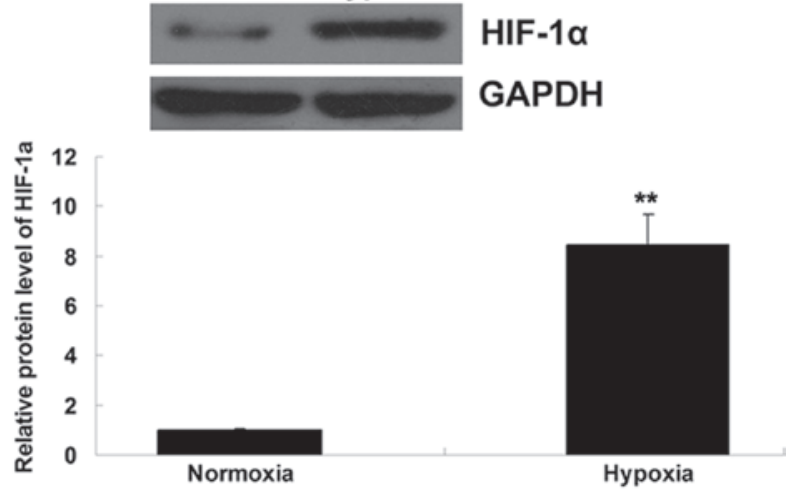

Figure 2. (A) Polymerase chain reaction analysis was performed to examine the mRNA expression of HIF-1 $\alpha$ in EPCs cultured under normoxia or hypoxia. GAPDH was used as an internal reference. ${ }^{* *} \mathrm{P}<0.01$ vs. normoxia. (B) Western blot analysis was performed to examine the protein expression of HIF-1 $\alpha$ in EPCs cultured under normoxia or hypoxia. GAPDH was used as an internal reference. ${ }^{* *} \mathrm{P}<0.01$ vs. normoxia. Values are expressed as the mean \pm standard deviation. HIF, hypoxia-inducible factor; EPC, endothelial progenitor cell.

A

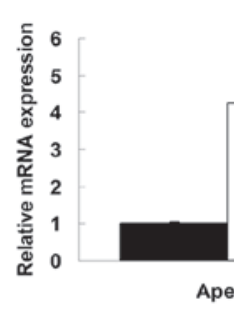

C

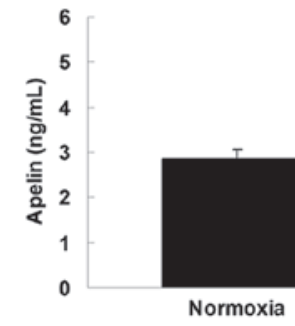

normoxia $\square$ Hypoxia
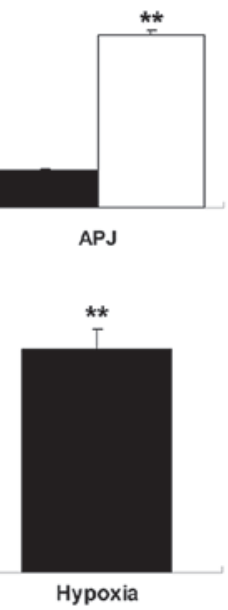

B
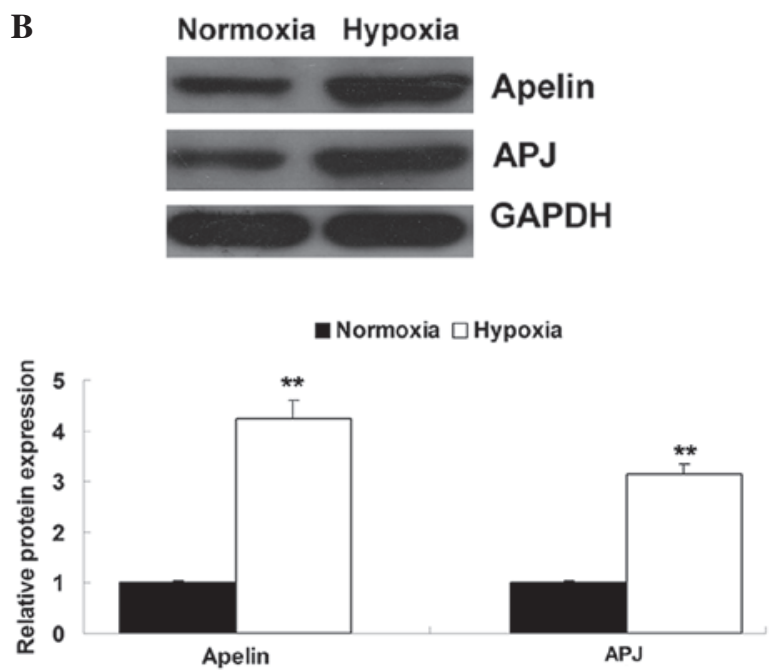

Figure 3. (A) Polymerase chain reaction was performed to examine the mRNA expression of apelin and APJ in EPCs cultured under normoxia or hypoxia. GAPDH was used as an internal reference. ${ }^{* *} \mathrm{P}<0.01$ vs. normoxia. (B) Western blot analysis was performed to examine the protein expression of apelin and APJ in EPCs cultured under normoxia or hypoxia. GAPDH was used as an internal reference. ${ }^{* *} \mathrm{P}<0.01$ vs. normoxia. (C) ELISA was performed to examine the secretion levels of apelin in EPCs cultured under normoxia or hypoxia. ${ }^{* *} \mathrm{P}<0.01$ vs. normoxia. Values are expressed as the mean \pm standard deviation. EPC, endothelial progenitor cell.

(Pierce Chemical, Rockford, IL, USA) was used to perform chemiluminescent detection. The relative expression of protein was determined by Image-Pro plus software 6.0 (Media Cybernetics, Inc., Rockville, MD, USA) represented as the density ratio versus GAPDH. The film was purchased from Kodak (Tokyo, Japan).

Statistical analysis. Values are expressed as the mean \pm standard deviation of three independent experiments. Statistical analysis of differences between values was performed using one-way analysis of variance with SPSS 17 software (SPSS, Inc., Chicago, IL, USA). $\mathrm{P}<0.05$ was considered to indicate a statistically significant difference between values.

\section{Results}

Hypoxia promotes EPC proliferation. First, the proliferation of EPCs was determined under hypoxia and normoxia by using an MTT assay. As shown in Fig. 1, after culturing under hypoxia for $6,12,24$ and $48 \mathrm{~h}$, the relative proliferation of EPCs was notably upregulated compared with that of EPCs cultured in normoxia. These findings indicated that hypoxia promoted EPC proliferation.

Hypoxia induces upregulation of HIF-1 $\alpha$ and apelin/APJ signaling. The expression levels of HIF-1 $\alpha$ were determined in each group, and it was found that hypoxia induced upregulation of HIF-1 $\alpha$ mRNA and protein expression in EPCs (Fig. 2A and B). As apelin and APJ are known to be regulated by HIF-1 $\alpha$, their expression levels in EPCs cultured in hypoxia or normoxia were then determined. As shown in Fig. 3A and B, the mRNA and protein levels of apelin and APJ were significantly upregulated in EPCs cultured in hypoxia. In addition, ELISA was performed to examine the secretion of apelin. As demonstrated in Fig. 3C, the secretion levels of apelin in hypoxia-treated EPCs were also upregulated 
A
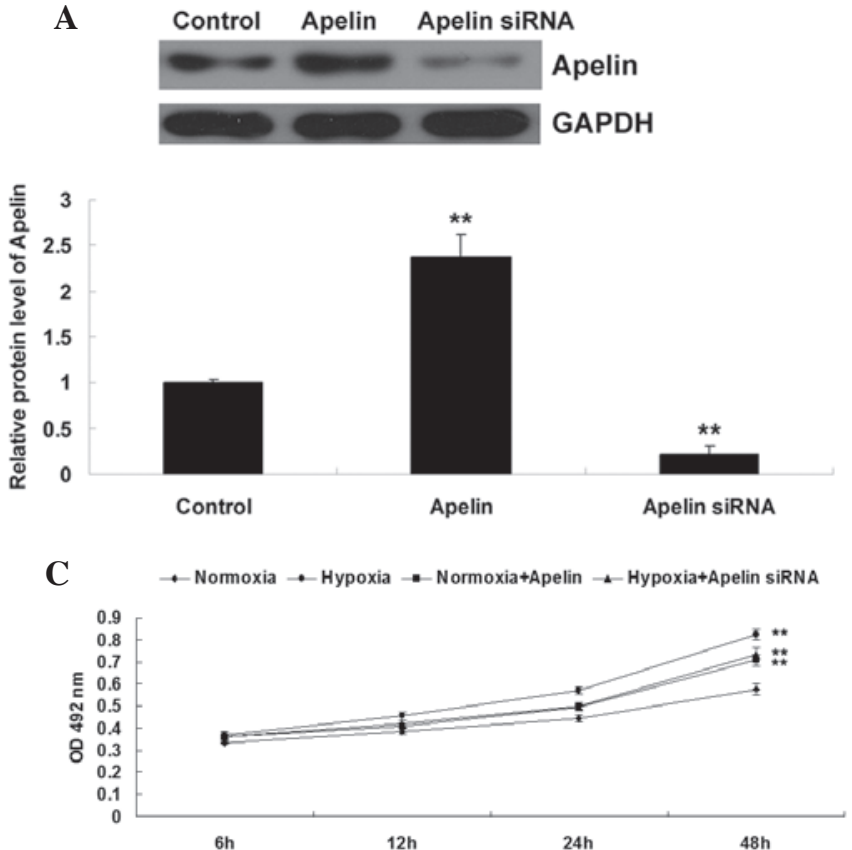

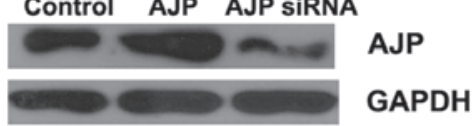

D
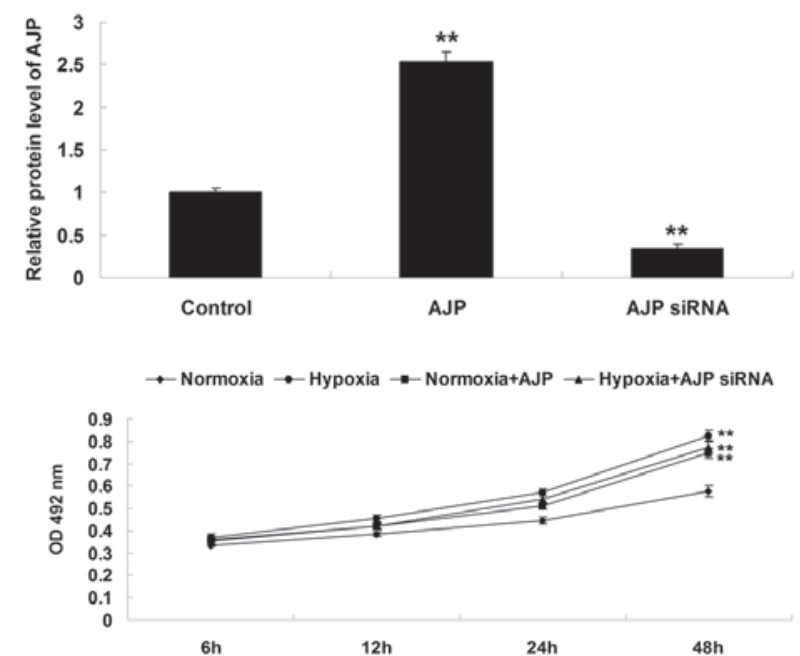

Figure 4. (A) Western blot analysis was performed to examine the protein expression of apelin in EPCs transfected with apelin plasmid or apelin siRNA, respectively. (B) Western blot analysis was performed to examine the protein expression of APJ in EPCs transfected with APJ plasmid or APJ siRNA, respectively. GAPDH was used as an internal reference. Control cells were not transfected. ${ }^{* *} \mathrm{P}<0.01$ vs. control. (C) An MTT assay was performed to determine the cell proliferation of EPCs in each group at 6,12,24 and 48 h. Groups: Normoxia, EPCs cultured under normoxia; Hypoxia, EPCs cultured under hypoxia; Normoxia + Apelin, EPCs transfected with apelin plasmid and cultured under normoxia; Hypoxia + Apelin siRNA, EPCs transfected with apelin siRNA and cultured under hypoxia. ${ }^{* *} \mathrm{P}<0.01$, vs. normoxia. (D) An MTT assay was performed to determine the cell proliferation of EPCs in each group at 6, 12, 24 and 48 h. Groups: Normoxia, EPCs cultured under normoxia; Hypoxia, EPCs cultured under hypoxia; Normoxia + APJ, EPCs transfected with APJ plasmid and cultured under normoxia; Hypoxia + APJ siRNA, EPCs transfected with APJ siRNA and cultured under hypoxia. "* $<<0.01$, vs. normoxia. Values are expressed as the mean \pm standard deviation. EPC, endothelial progenitor cell; siRNA, small interfering RNA; OD, optical density.

compared to those in EPCs cultured under normoxia. These findings suggested that HIF-1 $\alpha$ as well as its downstream apelin/APJ signaling were upregulated in hypoxia-treated EPCs.

Apelin/APJ signaling has a role in hypoxia-induced EPC proliferation. The detailed role of apelin/APJ signaling in hypoxia-induced EPCs proliferation was investigated. Four groups were established: The normoxia group as a control, a hypoxia group, a normoxia + apelin group and a hypoxia + apelin siRNA group. After transfection of the EPCs with apelin plasmid or siRNA, respectively, the expression levels of apelin were determined using western blotting, and the results showed satisfactory transfection efficiency (Fig. 4A). After that, the role of APJ in the regulation of hypoxia-induced EPC proliferation was investigated, for which four groups were established: A normoxia group as the control, a hypoxia group, a hormoxia + APJ group and a hypoxia + APJ siRNA group. After transfection of EPCs with APJ plasmid or siRNA, respectively, the expression levels of APJ were determined using western blotting, and the results showed satisfactory transfection efficiency (Fig. 4B). Subsequently, EPC proliferation was determined in each group by using the MTT assay. As shown in Fig. 4C, the proliferation of EPCs in the normoxia + apelin group was higher than that in the normoxia group, and the proliferation of EPCs in the hypoxia + apelin siRNA group was lower than that in the hypoxia group. Furthermore, as shown in Fig. 4D, the proliferation of EPCs in the normoxia + APJ group was

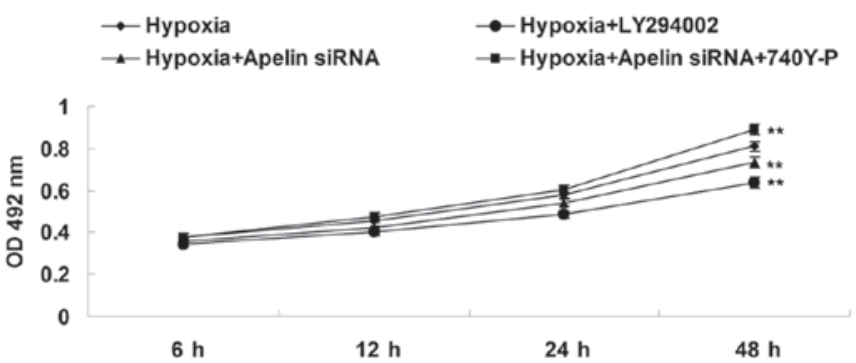

Figure 5. An MTT assay was performed to determine the cell proliferation of EPCs in each group at 6, 12, 24 and 48 h. Groups: Hypoxia, EPCs cultured under hypoxia; Hypoxia + Apelin siRNA, EPCs transfected with apelin siRNA and cultured under hypoxia; Hypoxia + LY294002, EPCs treated with LY294002 and cultured under hypoxia; Hypoxia + Apelin siRNA + 740Y-P, EPCs transfected with apelin siRNA, treated with 740Y-P and cultured under hypoxia. ${ }^{* *} \mathrm{P}<0.01$, vs. hypoxia. Values are expressed as the mean \pm standard deviation. OD, optical density; siRNA, small interfering RNA; EPC, endothelial progenitor cell.

higher than that in the normoxia group, and the proliferation of EPCs in the hypoxia + APJ siRNA group was lower than that in the hypoxia group. These findings suggested that apelin/APJ signaling has a role in hypoxia-induced EPC proliferation.

PI3K/Akt pathway is involved in apelin/APJ-mediated hypoxia-induced EPC proliferation. The present study further determined the underlying molecular mechanism by which apelin/APJ mediated EPC proliferation under hypoxia. To determine whether the PI3K/Akt pathway was 
involved in the apelin/APJ-mediated EPC proliferation, PI3K inhibitor LY294002 and PI3K agonist 740Y-P were used. Four groups were set as follows: The hypoxia group, the hypoxia + LY294002 group, the hypoxia + apelin siRNA group and the hypoxia + apelin siRNA + 740Y-P group. As shown in Fig. 5, the proliferation of EPCs in the hypoxia + LY294002 group was lower than that in the hypoxia group, suggesting that inhibition of PI3K/Akt signaling suppressed hypoxia-induced EPC proliferation. In addition, the proliferation of EPCs in the hypoxia + apelin siRNA + 740Y-P group was higher than that in the hypoxia + apelin siRNA group, suggesting that upregulation of PI3K/Akt signaling reversed the inhibitory effect of apelin siRNA on hypoxia-induced EPCs proliferation. These results suggested that the PI3K/Akt pathway acts as a downstream effector of apelin/APJ signaling in EPCs, and is involved in the apelin/APJ-mediated EPC proliferation under hypoxia.

\section{Discussion}

The present study showed that hypoxia induced upregulation of HIF-1 $\alpha$ and apelin/APJ signaling in EPCs. Increased apelin/APJ further activated PI3K/Akt signaling, which was involved in the upregulation of EPC proliferation. The findings of the present study highlighted the importance of apelin/APJ signaling in the regulation of EPC proliferation under hypoxia.

The critical role of EPCs in angiogenesis under hypoxia/ischemia has been widely revealed. It has been well established that hypoxia/ischemia triggers EPCs to migrate from the bone marrow into the peripheral blood (14). After migration to the site of hypoxic/ischemic tissues, EPCs can differentiate into ECs and participate in the formation of novel vessels. HIF-1 $\alpha$ is a key determinant of oxygen-dependent gene regulation in angiogenesis, which has been shown to be involved in EPC proliferation and differentiation $(8,15)$. For instance, several studies found that inhibition of HIF-1 $\alpha$ was able to inhibit the expression of vascular endothelial growth factor (VEGF), VEGF receptor 2, endothelial nitric oxide synthase as well as NO production, and suppress the differentiation of EPCs to ECs $(8,15)$. On the contrary, overexpression of HIF-1 $\alpha$ was reported to promote the differentiation of EPCs to ECs (16). The present study showed that under hypoxia, the expression of HIF-1 $\alpha$ was significantly upregulated in EPCs, consistent with the results of other studies $(4,5)$. Furthermore, the present study found that the expression of apelin was upregulated under hypoxia in EPCs. In fact, the association between HIF-1 $\alpha$ and apelin has been elucidated by previous studies. Ronkainen et al (17) showed that the upregulation of apelin under hypoxia was abolished by the HIF-inhibitory PAS protein in cardiomyocytes, suggesting that the expression of apelin was regulated by hypoxia in cardiac myocytes via the HIF pathway. Furthermore, Glassford et al (18) showed that HIF-1 $\alpha$ regulated hypoxia-induced expression of apelin in adipocytes. However, to the best of our knowledge, the association between HIF-1 $\alpha$ and apelin in EPCs has never been reported. As the results of the present study showed that hypoxia induced the upregulation of HIF-1 $\alpha$ as well as apelin, it was hypothesized that the upregulation of HIF-1 $\alpha$ induced by hypoxia may further promote the expression of apelin in EPCs.
Apelin/APJ has been found to be involved in a wide range of physiological and pathological functions in the cardiovascular system (19). For instance, apelin has a crucial role in the regulation of cell proliferation in vascular smooth muscle cells (VSMCs) (19). Moreover, inhibition of apelin expression markedly inhibited EC proliferation in pathological retinal angiogenesis (20). Furthermore, apelin/APJ was found to increase angiogenesis and improve cardiac functional recovery post-myocardial infarction (21). These findings suggested that Apelin has a key role in the regulation of cell proliferation in the cardiovascular system. Recently, apelin was reported to be involved in the mobilization of EPCs after acute myocardial infarction (12). However, whether apelin mediates EPCs proliferation has never been investigated, to the best of our knowledge. The present study showed that siRNA-mediated inhibition of apelin/APJ signaling markedly attenuated EPC proliferation induced by hypoxia, indicating that apelin/APJ signaling acts as a downstream regulator in hypoxia-induced EPC proliferation.

Furthermore, it was found that inhibition of PI3K/Akt signaling suppressed hypoxia-induced EPC proliferation, while activation of PI3K/Akt signaling reversed the inhibitory effect of apelin/APJ signaling knockdown on hypoxia-induced EPC proliferation. These results confirmed that PI3K/Akt signaling was the downstream effector of apelin/APJ signaling in the regulation of EPC proliferation induced by hypoxia. Kleinz and Baxter (22) showed that the protective effect of apelin on myocardial reperfusion injury was independent of PI3K/Akt signaling. However, Tao et al (23) reported that apelin protected the heart against ischemia-reperfusion injury through activation of PI3K/Akt signaling, via inhibition of endoplasmic reticulum stress-dependent apoptosis activation. Moreover, another study also showed that apelin protected against ischemic cardiomyocyte apoptosis via activation of the PI3K/Akt pathway (24). Furthermore, Liu et al (25) showed that apelin was able to promote VSMC proliferation through activation of PI3K/Akt signaling. In fact, most studies demonstrated that PI3K/Akt signaling acted as a downstream effector of apelin, consistent with the findings of the present study.

In conclusion, the present study reported that hypoxia enhanced the expression of HIF-1 $\alpha$, which led to the upregulation of apelin/APJ signaling, and further activated the downstream PI3K/Akt signaling, an important promoter of EPC proliferation. Therefore, apelin/APJ may serve as a potential target for the prevention of hypoxic/ischemic injury in the cardiovascular system.

\section{References}

1. Ganguly R, Lytwyn MS and Pierce GN: Differential effects of trans and polyunsaturated fatty acids on ischemia/reperfusion injury and its associated cardiovascular disease states. Curr Pharm Des, 2013.

2. Kalogeris T, Baines CP, Krenz M and Korthuis RJ: Cell biology of ischemia/reperfusion injury. Int Rev Cell Mol Biol 298: 229-317, 2012.

3. Schröder K, Kohnen A, Aicher A, et al: NADPH oxidase Nox2 is required for hypoxia-induced mobilization of endothelial progenitor cells. Circ Res 105: 537-544, 2009.

4. Simons D, Grieb G, Hristov M, et al: Hypoxia-induced endothelial secretion of macrophage migration inhibitory factor and role in endothelial progenitor cell recruitment. J Cell Mol Med 15: 668-678, 2011. 
5. Lee SH, Lee JH, Yoo SY, Hur J, Kim HS and Kwon SM: Hypoxia inhibits cellular senescence to restore the therapeutic potential of old human endothelial progenitor cells via the hypoxia-inducible factor-1alpha-TWIST-p21 axis. Arterioscler Thromb Vasc Biol 33: 2407-2414, 2013.

6. Dai T, Zheng H and Fu GS: Hypoxia confers protection against apoptosis via the PI3K/Akt pathway in endothelial progenitor cells. Acta Pharmacol Sin 29: 1425-1431, 2008.

7. Zhu G,Tang Y,Geng N, etal:HIF-alpha/MIFand NF-kappaB/IL-6 axes contribute to the recruitment of $\mathrm{CD} 11 \mathrm{~b}+\mathrm{Gr}-1+$ myeloid cells in hypoxic microenvironment of HNSCC. Neoplasia 16: 168-179, 2014.

8. Jiang M, Wang CQ, Wang BY and Huang DJ: Inhibitory effect of siRNA targeting HIF-1alpha on differentiation of peripheral blood endothelial progenitor cells. Ai Zheng 24: 1293-1300, 2005 (In Chinese)

9. Hashimoto T, Kihara M, Imai N, et al: Requirement of apelin-apelin receptor system for oxidative stress-linked atherosclerosis. Am J Pathol 171: 1705-1712, 2007.

10. Bertrand C, Pignalosa A, Wanecq E, et al: Effects of dietary eicosapentaenoic acid (EPA) supplementation in high-fat fed mice on lipid metabolism and apelin/APJ system in skeletal muscle. PLoS One 8: e78874, 2013.

11. O'Carroll AM, Lolait SJ, Harris LE and Pope GR: The apelin receptor APJ: journey from an orphan to a multifaceted regulator of homeostasis. J Endocrinol 219: R13-R35, 2013.

12. Ye J, Ni P, Kang L and Xu B: Apelin and vascular endothelial growth factor are associated with mobilization of endothelial progenitor cells after acute myocardial infarction. J Biomed Res 26: 400-409, 2012.

13. Wang W, McKinnie SM, Patel VB, et al: Loss of Apelin exacerbates myocardial infarction adverse remodeling and ischemia-reperfusion injury: therapeutic potential of synthetic Apelin analogues. J Am Heart Assoc 2: e000249, 2013.

14. Machalińska A: The role of circulating endothelial progenitor cells in the development of vascular retinal diseases. Klin Oczna 115: 158-162, 2013 (In Polish).
15. Jiang M, Wang B, Wang C, et al: Inhibition of hypoxia-inducible factor- $1 \alpha$ and endothelial progenitor cell differentiation by adenoviral transfer of small interfering RNA in vitro. J Vasc Res 43: 511-521, 2006

16. Jiang M, Wang CQ, Wang BY, He B, Shao Q and Huang DJ: Overexpression of hypoxia inducible factor- $1 \alpha$ (HIF-1 $\alpha$ ) promotes the differentiation of endothelial progenitor cell ex vivo. Zhongguo Shi Yan Xue Ye Xue Za Zhi 14: 565-570, 2006 (In Chinese).

17. Ronkainen VP, Ronkainen JJ, Hänninen SL, et al: Hypoxia inducible factor regulates the cardiac expression and secretion of apelin. FASEB J 21: 1821-1830, 2007.

18. Glassford AJ, Yue P, Sheikh AY, et al: HIF-1 regulates hypoxiaand insulin-induced expression of apelin in adipocytes. Am J Physiol Endocrinol Metab 293: E1590-E1596, 2007.

19. Lv D, Li H and Chen L: Apelin and APJ, a novel critical factor and therapeutic target for atherosclerosis. Acta Biochim Biophys Sin (Shanghai) 45: 527-533, 2013.

20. Kasai A, Ishimaru Y, Higashino K, et al: Inhibition of apelin expression switches endothelial cells from proliferative to mature state in pathological retinal angiogenesis. Angiogenesis 16: 723-734, 2013.

21. Li L, Zeng H and Chen JX: Apelin-13 increases myocardial progenitor cells and improves repair postmyocardial infarction. Am J Physiol Heart Circ Physiol 303: H605-H618, 2012.

22. Kleinz MJ and Baxter GF: Apelin reduces myocardial reperfusion injury independently of PI3K/Akt and P70S6 kinase. Regul Pept 146: 271-277, 2008.

23. Tao J, Zhu W, Li Y, et al: Apelin-13 protects the heart against ischemia-reperfusion injury through inhibition of ER-dependent apoptotic pathways in a time-dependent fashion. Am J Physiol Heart Circ Physiol 301: H1471-H1486, 2011.

24. Zhang Z, Yu B and Tao GZ: Apelin protects against cardiomyocyte apoptosis induced by glucose deprivation. Chin Med J (Engl) 122: 2360-2365, 2009.

25. Liu C, Su T, Li F, et al: PI3K/Akt signaling transduction pathway is involved in rat vascular smooth muscle cell proliferation induced by apelin-13. Acta Biochim Biophys Sin (Shanghai) 42: 396-402, 2010. 